\title{
Asynchronous cooperative communication systems: A survey on signal designs
}

\author{
WANG HuiMing ${ }^{1,2}$ \& XIA XiangGen ${ }^{3 *}$ \\ ${ }^{1}$ School of Electronic and Information Engineering, Xi'an Jiaotong University, \\ $X i$ 'an 710049, China; \\ ${ }^{2}$ State Key Laboratory of Integrated Services Networks, Xidian University, Xi'an 710049, China; \\ ${ }^{3}$ Department of Electrical and Computer Engineering, University of Delaware, \\ Newark, DE 19716, USA
}

Received February 24, 2011; accepted May 18, 2011; published online June 24, 2011

\begin{abstract}
Cooperative communications is a promising technique for future high speed wireless communications. These systems may be formulated as virtual multi-input multi-output (MIMO) systems where spatial/cooperetive diversity is a key advantage. However, different from MIMO systems, one of the major challenges for cooperative communications systems is that the cooperative transmissions in cooperative systems may be neither time nor frequency synchronized, since the transmissions are from multiple cooperative nodes at different locations. The existing signal designs for co-located MIMO systems may not be able to collect the cooperative diversity in cooperative communications systems. This paper gives an overview of recent research efforts on combating the time and frequency asynchronism of the cooperative communication network. We focus on the signal designs (or space-time codings/modulations) to achieve full cooperative diversity, and summarize some of the resent distributed space-timing coding and space-frequency coding techniques to combat timing errors and frequency offsets, and in the meantime to achieve full cooperative diversity, in both one-way and two-way cooperative networks.
\end{abstract}

Keywords cooperative communications, time and frequency offsets, space-time coding, space-frequency codes, full cooperative diversity, linear receiver

Citation Wang H M, Xia X G. Asynchronous cooperative communication systems: A survey on signal designs. Sci China Inf Sci, 2011, 54: 1547-1561, doi: 10.1007/s11432-011-4306-8

\section{Introduction}

One basic challenge for future wireless communication systems is the contradiction between fast growing demands for large capacity high speed wireless communications and limited electromagnetic wave frequency spectrum. The multiple-input multiple-output (MIMO) technique [1, 2] with space-time coding (STC) [3-6] has been recognized as a promising scheme for the next-generation of broadband wireless communication systems. It is because of its capability of providing spatial diversity to combat fading in wireless channels and increasing capacity without additional power consumption or bandwidth expansion. However, all these improvements come at the cost of multiple radio frequency (RF) front ends at both the transmitter and the receiver. The size of the mobile devices and the requirement on distance between

*Corresponding author (email: xianggen@gmail.com) 
antennas (to make the channel fading between antenna pairs uncorrelated) may limit the number of antennas that can be deployed. How to exploit the spatial benefits by wireless terminals with only single antenna becomes a natural question. Cooperative communications technology [7-12] may have provided an answer to this question.

In cooperative communications, multiple terminals in a wireless network are capable of working together: one terminal can help others to transmit their desired signals. As such, both the spatial links of itself and the helper can be exploited and as a result, multiple single-antenna terminals can share their antennas to create a virtual antenna array. Meanwhile, due to its distributed nature, independently faded channel could be guaranteed to provide spatial degree-of-freedom. Through cooperation, it becomes possible to exploit the spatial resource of the traditional MIMO techniques without the need to equip each terminal with multiple antennas. Therefore, it can be considered as a distributed MIMO system, while the traditional MIMO can be named as co-located MIMO. Since the early studies by Sendonaris et al. [7] and Laneman et al. [8], cooperative communications techniques have attracted tremendous interest over the recent years, both in academy and industry. Distributed space-time coding (DSTC), i.e., the distributed counterpart of space-time coding in co-located MIMO, has become an efficient technique to provide spatial diversity for cooperative communication systems [9-12]. On one hand, cooperation is embedded into cellular mobile communications system and new cooperative stations may be built in a cellular system [13]; on the other hand, cooperative communications technique is adopted in the physical layer of ad hoc networks and wireless sensor networks (WSN) [14, 15].

Compared with the co-located MIMO systems, the most distinctive characteristic of cooperative communications systems is its distributed nature: multiple terminals in cooperation are spatially separated in several different physical locations. Thus, some new problems that do not exist in co-located MIMO systems have merged: for example,

1) Signal coordination problem: Whether and when a terminal participates in the cooperation, and what a terminal should transmit.

2) Time asynchronism problem: Multiple cooperative terminals have different distances to the destination terminal and thus the cooperative transmission may be time-asynchronous.

3) Frequency asynchronism problem: Each cooperative terminal has its own oscillator and thus the cooperative transmission is frequency-asynchronous.

Although centralized high layer control protocols can be used to solve some of these problems, for example, a central station can be set to guarantee the signal coordination, and synchronize the cooperative terminals, a large amount of extra control signals are required, which may reduce the benefits of cooperation greatly. On the other hand, it may be difficult for wireless networks without infrastructure like an ad hoc network or a WSN to have a central control station. Also, these networks are generally with high dynamics, where nodes frequently join and leave the network, which further increases the overhead significantly. Therefore, people take great interest in dealing with these problems by physical layer techniques, such as coding/modulation and advanced signal processing.

In this paper, we focus on the last two problems, i.e., time and frequency asynchronism, and overview some of the research efforts on this topic in recent years. We will summarize various distributed STCs and equalization methods that have been proposed for cooperative nodes and destination node to combat time and frequency asynchronism, respectively, aiming at achieving as much diversity gain as possible. In these works, it is often assumed that the cooperative network has already been coordinated. We do not cover the literatures from an information theory point of view, for example [16]. We also assume that all the channel parameters, including the fading coefficients, the time and frequency offsets are perfectly obtained at the destination node by some estimation algorithms, such as those proposed in [17-21].

The paper is organized as follows. In Section 2, we present the system model of one-way and two-way cooperative networks under time and frequency asynchronism. In Section 3 and Section 4, various recent literatures addressing the time and frequency asynchronism of one-way and two-way cooperative networks are reviewed, respectively. Finally, in Section 5, conclusions are drawn and some future problems are proposed. 


\section{Asynchronous cooperative system model}

In this section, we give the asynchronous cooperative system model with time and frequency offsets. We consider both one-way and two-way cooperative networks. In a one-way network, there is one source and one destination, and the signal transmission is unidirectional, from the source to the destination. A two-way network includes two terminals acting as both source and destination. Two terminals want to exchange information and the signal transmissions are bi-directional. In both cases, we assume that each node in the network is only equipped with a single antenna and subject to the half-duplex constraint, i.e., they cannot transmit and receive simultaneously. Unless otherwise stated, the channels are undergoing flat fading, although some of the techniques, such as the orthogonal frequency division multiplexing (OFDM) techniques, overviewed in this paper have been extended to frequency-selective fading channels ${ }^{1)}$. We also assume that there is no direct connection between source and destination (or two source terminals) due to shadowing or too large distance.

\subsection{One-way cooperative network}

A general one-way cooperative network with one source node $\mathbb{S}$, one destination node $\mathbb{D}$ and $R$ cooperative nodes $\mathbb{R}_{r}, r=1,2, \ldots, R$, between the source and the destination is shown in Figure 1 . The cooperation contains two phases [9]. In the first phase, the source broadcasts the information sequence to potential cooperative nodes. Under decode-and-forward (DF) protocol, at each potential cooperative node, the received signals are decoded. To guarantee the success of cooperation, only the cooperative node that correctly decodes the received signals will become an active cooperative node to participate in the cooperative transmission in the second phase. A simple manner to do this is to use an error detection code such as cyclic redundancy check (CRC) in the source [10]. In the beginning of the second phase, the eligible cooperative nodes re-map the decoded information bits into symbols. Before they are transmitted to the destination node, various coding schemes, from the simple repetition code to many elaborately designed distributed space-time coding schemes, are proposed in the literature to extract spatial diversity. The coded signals are then transmitted to the destination. If amplify-and-forward (AF) protocol is adopted, the source information symbols are not decoded in the cooperative nodes. Only a simple amplification (or, more general, a linear operation) is taken by each cooperative node and then these linearly transformed signals are transmitted from cooperative nodes.

1) Time Asynchronism: Since the cooperative nodes are in different physical locations, the time delay from the source to each cooperative node in the first phase is different. In the second phase, the retransmitted signal from each cooperative node to the destination will also undergo different time delays. Therefore, the received signals at the destination may not align, and the time asynchronism is the accumulation of both phases.

Denote the delays from source to cooperative node $r$ by $\tau_{s, r}$ and those from $r$ to destination by $\tau_{r, d}$. Also assume that the processing delays at the cooperative nodes are the same so that they can be omitted. Then the total delay of the $r$-th link is $\tau_{r}=\tau_{s, r}+\tau_{r, d}$. Without loss of generality, we may assume $0=\tau_{1}<\tau_{2}<\cdots<\tau_{R}$. Assume that a distributed space-time block code (DSTBC) is adopted by the cooperative nodes, which can be expressed as

$$
\boldsymbol{X}=\left[\boldsymbol{x}_{1}, \boldsymbol{x}_{2}, \ldots, \boldsymbol{x}_{R}\right]=\left[\begin{array}{cccc}
x_{1,1} & x_{1,2} & \cdots & x_{1, R} \\
x_{2,1} & x_{2,2} & \cdots & x_{2, R} \\
\vdots & \vdots & \ddots & \vdots \\
x_{L, 1} & x_{L, 2} & \cdots & x_{L, R}
\end{array}\right]
$$

where $\boldsymbol{x}_{r}$ is transmitted by cooperative node $r, r=1,2, \ldots, R$, and $L$ is the length of a DSTBC code block. If the system is perfectly synchronized, then the received baseband signal at the destination can

1) Here, we do not assume a particular model statistics of the channel coefficients, such as Rayleigh or Rice [22]. The diversity gain analysis applies to both Rayleigh and Rice fading channels similar to MIMO systems 


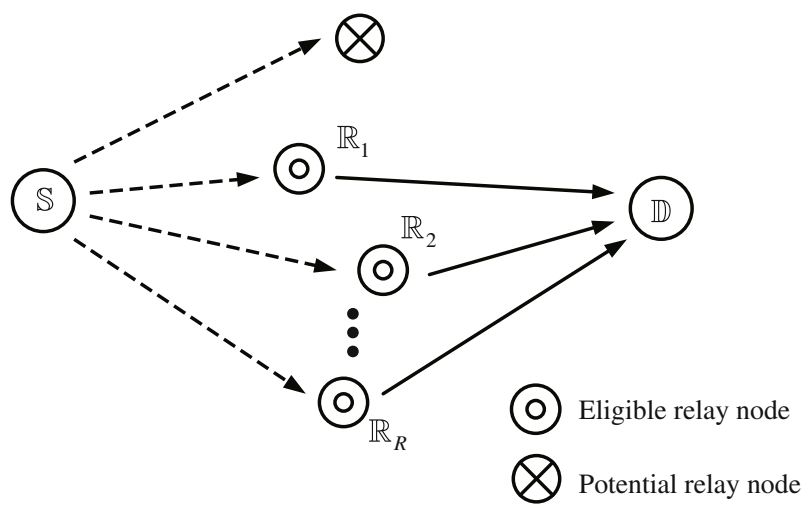

Figure 1 One-way cooperative network model, where a dashed line is a transmission in the first phase and a solid line is a transmission in the second phase.

be written as

$$
\boldsymbol{y}_{d}=\boldsymbol{X}_{\tau} \boldsymbol{h}+\boldsymbol{n}_{d}
$$

where $\boldsymbol{h}=\left[h_{1}, h_{2}, \ldots, h_{R}\right]^{\mathrm{T}}$ is the channel coefficient vector. However, when the time delays exist, the equivalent DSTC code matrix becomes

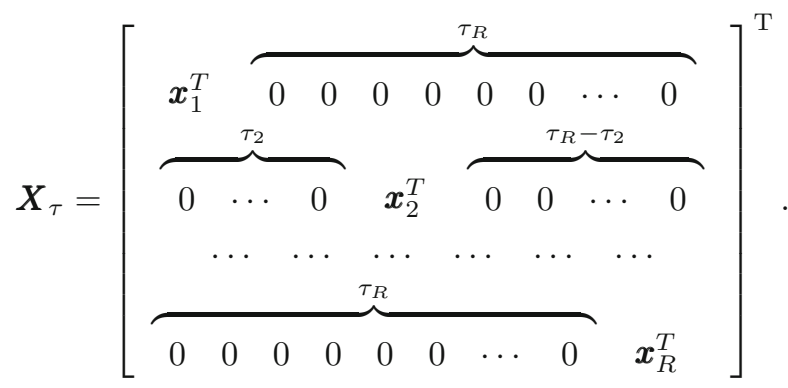

Here, we only consider the case where the delays are multiples of symbol duration for convenience. And fractional delays of symbol duration can be similarly discussed. We can see that due to the time asynchronism, the structure of elaborately designed DSTBC is destroyed, which will decrease the achieveable diversity gain.

2) Frequency asynchronism: Since the source, destination and each cooperative node have their respective oscillators, it is hard to lock them to exact the same frequency and as a result, there may exist a carrier frequency offset (CFO) between each pair of transmitter and receiver. In the first phase, there is only one CFO between each cooperative node and the source. The received baseband signal at cooperative node $r$ is

$$
y_{r}(n)=\mathrm{e}^{\mathrm{j} 2 \pi n T \triangle f_{r}^{\prime}} g_{r} x_{s}(n)+w_{r}(n), \quad n=0,1, \ldots, N-1,
$$

where $T$ is the symbol duration, $g_{r}, \triangle f_{r}^{\prime}$ and $w_{r}(r=1,2, \ldots, R)$ are the channel coefficient, the CFO and noise of the $r$-th cooperative node, respectively, and $x_{s}$ is the source information. So the cooperative node can estimate the CFO and then compensate for it completely, as can be done in single-input singleoutput (SISO) system, which has already been well addressed, see [17], for example. In the second phase, however, the destination faces $R$ CFOs, one from each cooperative node. The received baseband signal at the destination is

$$
y_{d}(n)=\sum_{r=1}^{R} \mathrm{e}^{\mathrm{j} 2 \pi n T \triangle f_{r}} h_{r} x_{r}(n)+w_{d}(n),
$$

where $h_{r}, \triangle f_{r}$ are the channel coefficient and CFO between cooperative $r$ and the destination. Because the multiple CFOs $\triangle f_{r}(r=1,2, \ldots, R)$ are generally different, it is hard for the destination node to compensate for them simultaneously. Consequently, the channels become time-varying from symbol to symbol, and STBCs for the conventional co-located MIMO systems may not be applied to achieve the spatial diversity. 


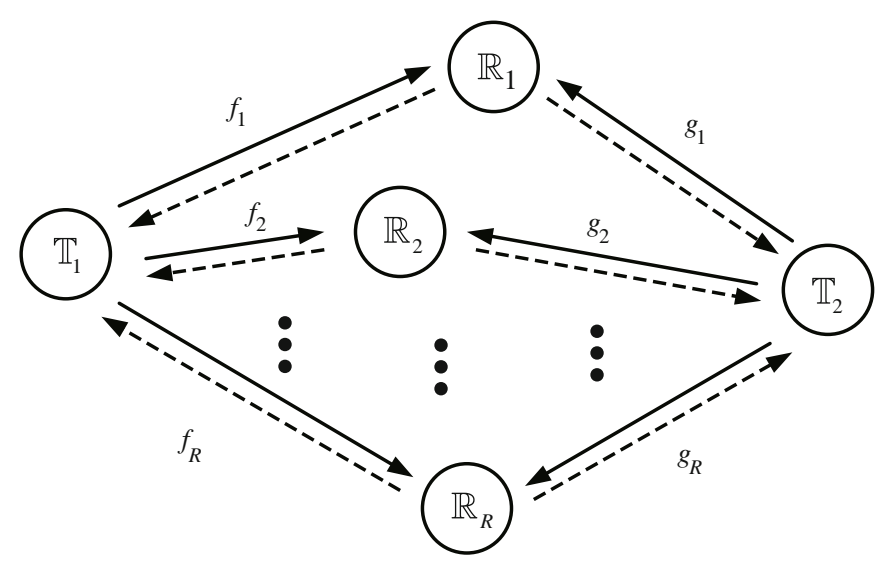

Figure 2 Two-way cooperative network model, where a solid line is a transmission in the first phase and a dashed line is a transmission in the second phase.

\subsection{Two-Way Cooperative Network}

A general two-way cooperative network with two terminal nodes $\mathbb{T}_{m}, m=1,2$ to exchange information through $R$ cooperative nodes $\mathbb{R}_{r}, r=1,2, \ldots, R$ is depicted in Figure 2 . If a similar protocol as in oneway cooperative network is exploited, then one round of information exchange requires four phases: in the first two phases, terminal $\mathbb{T}_{1}$ sends information to $\mathbb{T}_{2}$ through cooperative nodes and in the second two phases, terminal $\mathbb{T}_{2}$ sends information to $\mathbb{T}_{1}$. However, by using the so-called analog network coding (ANC), the whole procedure can be shortened to two phases, as shown in Figure 2. In the first phase, both $\mathbb{T}_{1}$ and $\mathbb{T}_{2}$ transmit their respective signals $\boldsymbol{s}_{1}$ and $\boldsymbol{s}_{2}$ simultaneously to all the cooperative nodes. Stack the received signal at the cooperative nodes into a vector $\boldsymbol{y}_{r}$, which is the mixed signal expressed as

$$
\boldsymbol{y}_{r}=\sqrt{P_{T_{1}}} f_{r} \boldsymbol{s}_{1}+\sqrt{P_{T_{2}}} g_{r} \boldsymbol{s}_{2}+\boldsymbol{n}_{r},
$$

where $P_{T_{m}}, m=1,2$, is the average transmit power of $\mathbb{T}_{m}$. In the second phase, a cooperative node processes the received mixed signals and then broadcast the so-obtained signal back to both $\mathbb{T}_{1}$ and $\mathbb{T}_{2}$. Assume that a cooperative node just multiplies the received signal by a scale factor $\alpha_{r}$ to satisfy the average transmit power constraint of $\mathbb{R}_{r}$ (generally, any linear or non-linear operation is possible). Then the receiving signal at terminal $\mathbb{T}_{1}$ is

$$
\boldsymbol{y}_{T_{1}}=\sum_{r=1}^{R} \alpha_{r} \sqrt{P_{T_{2}}} f_{r} g_{r} \boldsymbol{s}_{2}+\sum_{r=1}^{R} \alpha_{r} \sqrt{P_{T_{1}}} f_{r}^{2} \boldsymbol{s}_{1}+\boldsymbol{w}_{T_{1}}
$$

where $\boldsymbol{w}_{T_{1}}=\sum_{r=1}^{R} \alpha_{r} f_{r} \boldsymbol{n}_{r}+\boldsymbol{n}_{T_{1}} . \mathbb{T}_{2}$ has the similar receiving signal which is omitted here.

Although the final received signals at two terminals are still mixed ones, each terminal can subtract the backward signal of itself (the second term of (7)) and thus obtain the desired information data from the other terminal (the first term of (7)). As such, the information exchange can be completed in just two phases instead of four phases, thus greatly improving the bandwidth efficiency [23-27].

1) Time asynchronism: In a general two-way cooperative network with multiple cooperative nodes, there may exist two kinds of timing errors [28, 29]. The first kind is that in the first phase, the signals from two terminals may arrive at each cooperative node at different times. The second kind occurs in the second phase where the send-back signals from different cooperative nodes may arrive at one terminal node at different time, as illustrated in Figure 3. Denote the delay from $\mathbb{T}_{m}$ to $\mathbb{R}_{r}$ in the first phase by $\tau_{R_{r}, T_{m}}$, the delay from $\mathbb{R}_{r}$ to $\mathbb{T}_{m}$ in the second phase by $\tau_{T_{m}, R_{r}}, r=1, \ldots, R, m=1,2$, and only consider the case where the delay is a multiple of symbol duration. Denote $\tau_{\max }^{(1)}=\max \left\{\tau_{R_{r}, T_{m}}, r=1, \ldots, R, m=1,2\right\}$, $\delta_{R_{r}, T_{m}}=\tau_{\max }^{(1)}-\tau_{R_{r}, T_{m}}, \tau_{\max , T_{1}}^{(2)}=\max \left\{\tau_{T_{1}, R_{r}}, r=1, \ldots, R\right\}, \tau_{\max , T_{2}}^{(2)}=\max \left\{\tau_{T_{2}, R_{r}}, r=1, \ldots, R\right\}$, and $\delta_{T_{m}, R_{r}} \triangleq \tau_{\max , T_{m}}^{(2)}-\tau_{T_{m}, R_{r}}$ for $r=1, \ldots, R, m=1,2$. 


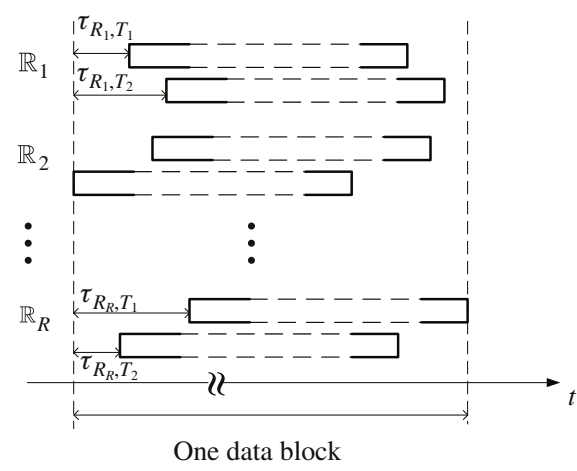

(a)

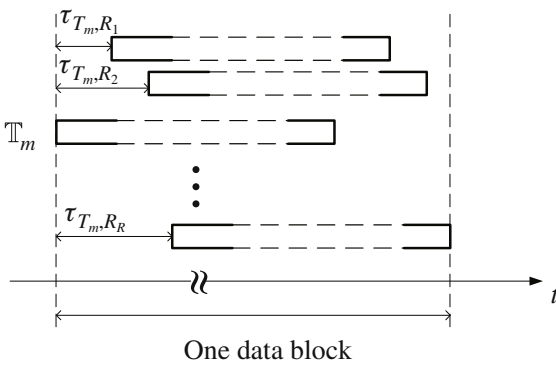

(b)

Figure 3 Two kinds of timing errors in a two-way cooperative network [29]. (a) The first kind of timing errors; (b) the second kind of timing errors, where $m=1,2$.

Taking these delays into account, if $L$ symbols are transmitted, the received mixed signals of $\mathbb{R}_{r}(6)$ at the end of the first phase will span $L^{\prime}=L+\tau_{\max }^{(1)}$ symbol intervals, which can be given by [29]

$$
\boldsymbol{y}_{r}^{\text {asyn }}=\sqrt{P_{T_{1}}} f_{r} \boldsymbol{D}_{R_{r}, T_{1}} \boldsymbol{s}_{1}+\sqrt{P_{T_{2}}} g_{r} \boldsymbol{D}_{R_{r}, T_{2}} \boldsymbol{s}_{2}+\boldsymbol{n}_{r},
$$

where $\boldsymbol{D}_{R_{r}, T_{m}} \triangleq\left[\begin{array}{lll}\mathbf{0}_{L \times \tau_{R_{r}, T_{m}}} & \boldsymbol{I}_{L} & \mathbf{0}_{L \times \delta_{R_{r}, T_{m}}}\end{array}\right]^{\mathrm{T}}, m=1,2$ are $L^{\prime} \times L$ matrices, named as timing error matrices. Similar to (7), when taking into account the delays, the block length of the received mixed signal $\boldsymbol{y}_{T_{1}}^{\text {asyn }}$ at $\mathbb{T}_{1}$ now becomes $L_{T_{1}}^{\prime \prime} \triangleq L^{\prime}+N-1+\tau_{\max , T_{1}}^{(2)}$, and the received signal can be written as

$$
\boldsymbol{y}_{T_{1}}^{\text {asyn }}=\sum_{r=1}^{R} \sqrt{P_{T_{2}}} f_{r} g_{r} \boldsymbol{D}_{T_{1}, R_{r}} \boldsymbol{T}_{r} \boldsymbol{D}_{R_{r}, T_{2}} \boldsymbol{s}_{2}+\sum_{r=1}^{R} \sqrt{P_{T_{1}}} f_{r}^{2} \boldsymbol{D}_{T_{1}, R_{r}} \boldsymbol{T}_{r} \boldsymbol{D}_{R_{r}, T_{1}} \boldsymbol{s}_{1}+\boldsymbol{w}_{T_{1}}^{a},
$$

where $\boldsymbol{T}_{r}$ is a matrix corresponding to some general linear operation taken at the cooperative nodes, $\boldsymbol{D}_{T_{m}, R_{r}} \triangleq\left[\begin{array}{lll}\mathbf{0}_{\left(L^{\prime}+N-1\right) \times \tau_{T_{m}, R_{r}}} & \boldsymbol{I}_{L^{\prime}+N-1} & \mathbf{0}_{\left(L^{\prime}+N-1\right) \times \delta_{T_{m}, R_{r}}}\end{array}\right]^{\mathrm{T}}$ are timing error matrices of size $L_{T_{m}}^{\prime \prime} \times$ $\left(L^{\prime}+N-1\right)$, and $\boldsymbol{w}_{T_{1}}^{a} \triangleq \sum_{r=1}^{R} f_{r} \boldsymbol{D}_{T_{1}, R_{r}} \boldsymbol{T}_{r} \boldsymbol{n}_{r}+\boldsymbol{n}_{T_{1}}$. Subtracting the second term of self-interference yields

$$
\boldsymbol{y}_{T_{1}}^{\text {asyn }}=\sum_{r=1}^{R} \alpha_{r} \sqrt{P_{T_{2}}} f_{r} g_{r} \boldsymbol{D}_{T_{1}, R_{r}} \boldsymbol{T}_{r} \boldsymbol{D}_{R_{r}, T_{2}} \boldsymbol{s}_{2}+\boldsymbol{w}_{T_{1}}^{a} \text {. }
$$

Let $\boldsymbol{c}_{r} \triangleq \boldsymbol{D}_{T_{1}, R_{r}} \boldsymbol{T}_{r} \boldsymbol{D}_{R_{r}, T_{2}} \boldsymbol{s}_{2}, \boldsymbol{X}_{t}^{a} \triangleq \sqrt{P_{T_{2}}}\left[\boldsymbol{c}_{1}, \boldsymbol{c}_{2}, \ldots, \boldsymbol{c}_{R}\right]$, and $\boldsymbol{h}_{e} \triangleq\left[f_{1} g_{1}, f_{2} g_{2}, \ldots, f_{R} g_{R}\right]^{\mathrm{T}}$. Then, (10) is immediately

$$
\boldsymbol{y}_{T_{1}}^{\text {asyn }}=\boldsymbol{X}_{t}^{a} \boldsymbol{h}_{e}+\boldsymbol{w}_{T_{1}}^{a} .
$$

By (11), after cancelling the self-data item, the data transmission from $\mathbb{T}_{2}$ to $\mathbb{T}_{1}$ can be viewed as a distributed space-time transmission system with equivalent STC matrix $\boldsymbol{X}_{t}^{a}$, vector channel $\boldsymbol{h}_{e}$ and equivalent Gaussian noise $\boldsymbol{w}_{T_{1}}^{a}$. Therefore, (11) can be called the equivalent STC model. Note that $\boldsymbol{X}_{t}^{a}$ is in a time-asynchronous manner, which can be written as [29]:

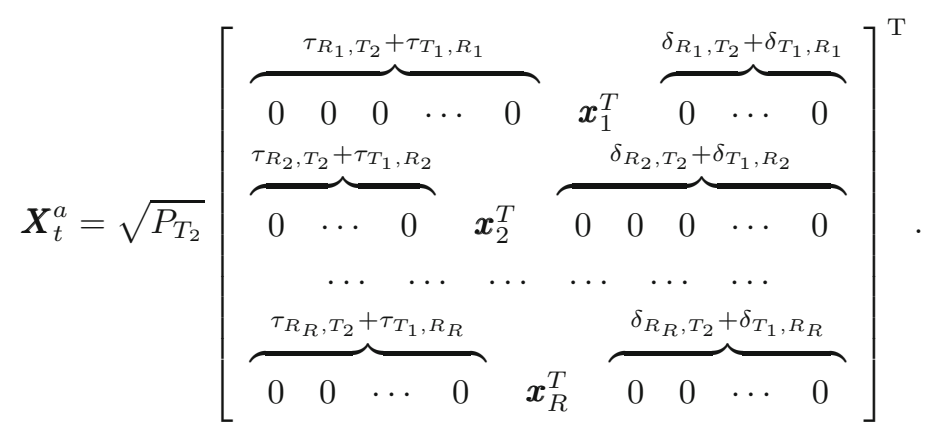

Note also that the noise term $\boldsymbol{w}_{T_{1}}^{a}$ combines both the noise at $\mathbb{T}_{1}$ and the amplified noise from cooperative nodes. If perfect synchronization is guaranteed, (11) is similar to the one-way cooperative network case 
based on AF protocol discussed in [12]. Under the time asynchronous case, we are now interested in the design of the code matrix $\boldsymbol{X}^{a}$ to achieve the potential cooperative diversity.

2) Frequency asynchronism: Different from the one-way cooperative network where multiple CFOs only exist in the second phase, in two-way cooperative network, frequency asynchronism occurs in both two phases. In the first phase, two CFOs exist for the received signal of each cooperative node, and in the second phase, each terminal obtains the mixed signal corrupted by $R$ CFOs, which is similar to that of the one-way cooperative network.

By doing similar operations as above, it is not hard to get the equivalent receiving model for $\mathbb{T}_{1}$ under the frequency asynchronism scenario

$$
\boldsymbol{y}_{T_{1}}^{\text {asyn }}=\boldsymbol{X}_{f}^{a} \boldsymbol{h}_{e}+\boldsymbol{w}_{T_{1}}^{a},
$$

where $\boldsymbol{X}_{f}^{a}=\sqrt{P_{T_{2}}}\left[\boldsymbol{c}_{1}, \boldsymbol{c}_{2}, \ldots, \boldsymbol{c}_{R}\right]$ with $\boldsymbol{c}_{r}=\boldsymbol{\Phi}_{1, r} \boldsymbol{T}_{r} \boldsymbol{\Phi}_{r, 2}, \boldsymbol{\Phi}_{1, r}$ is the frequency offset matrix from $\mathbb{R}_{r}$ to $\mathbb{T}_{1}$ and $\boldsymbol{\Phi}_{r, 2}$ is that from $\mathbb{T}_{2}$ to $\mathbb{R}_{r}, r=1,2, \ldots, R$. They both are diagonal matrices in the form of $\boldsymbol{\Phi}_{1, r}=\operatorname{diag}\left(1, \mathrm{e}^{\mathrm{j} \frac{2 \pi \phi_{1, r}}{L}}, \ldots, \mathrm{e}^{\mathrm{j} \frac{2 \pi(L-1) \phi_{1, r}}{L}}\right)$, and $\boldsymbol{\Phi}_{r, 2}=\operatorname{diag}\left(1, \mathrm{e}^{\mathrm{j} \frac{2 \pi \phi_{r, 2}}{L}}, \ldots, \mathrm{e}^{\mathrm{j} \frac{2 \pi(L-1) \phi_{r, 2}}{L}}\right)$, where $\phi_{i, j}$ is the CFO between $i$ and $j$.

\section{One-way cooperative network with asynchronous cooperative diversity}

Under the scenario that both time and frequency are perfectly synchronized, distributed space-time coding (DSTC) and distributed space-frequency coding (DSFC) are efficient ways to collect spatial diversity gain for cooperative networks, when the transmitters are lacking the channel state information (CSI). They can be considered as the distributed versions of their counterparts in co-located MIMO systems. However, when taking the time and frequency asynchronism into consideration, the existing DSTCs and DSFCs can not be simply applied. Both distributed transmission schemes at the cooperative nodes and receive algorithms at the destination need to be carefully re-investigated.

\subsection{Time asynchronism}

The approaches for combating the time asynchronism, like those for dealing with the intersymbol interference (ISI) channels, are generally classified into two categories: time-domain approaches and frequencydomain approaches. In time-domain approaches, various DSTCs without the requirement of time synchronization have been proposed [30-41]. In frequency-domain approaches, the basic idea is to treat the paths from cooperative nodes to the destination node as multipaths and then use OFDM technology [32, 42-47] to deal with the multipaths (or ISI). Using OFDM, cyclic prefix (CP) can be exploited to combat the time asynchronism, and the channels are now ISI-free and DSFCs are designed on the subcarriers of OFDM to collect spatial/cooperative diversity.

In [30], the idea of delay diversity code [48] for co-located MIMO system has been utilized. Different cooperative terminals transmit the same signals, but intentional delays are introduced in them. At the destination, a minimum mean square error (MMSE) criterion based fractional spaced decision feedback equalizer (DFE) is proposed to exploit the cooperative diversity. Although some diversity gain can be achieved in [30], full diversity order is not guaranteed. In [31, 32], it has been indicated that since time asynchronism makes the flat fading become frequency-selective fading, STBCs that apply in frequencyselective fading channels can be used to combat time asynchronism and to collect diversity gain. Both these two papers propose the distributed time reversal space-time block codes (D-TR-STBC), which is first proposed in [49]. However, the data rate of this scheme becomes very low when the number of cooperative nodes becomes large, due to the orthogonality of the code matrix. Using simply Alamouti STBC at the cooperative nods, the emphasis is put on the receiver design in [33]. An equalizer has been proposed: first cancel the interference components caused by timing misalignment in a decision feedback manner, and then implement a symbol by symbol maximum likelihood (ML) detection. It retains the low computational complexity of Alamouti code but only applies in the case of two cooperative nodes.

A systematic family of distributed space-time trellis codes (DSTTC) to collect the full cooperative diversity without the synchronicity assumption is first proposed in [34]. The basic idea is to design the 


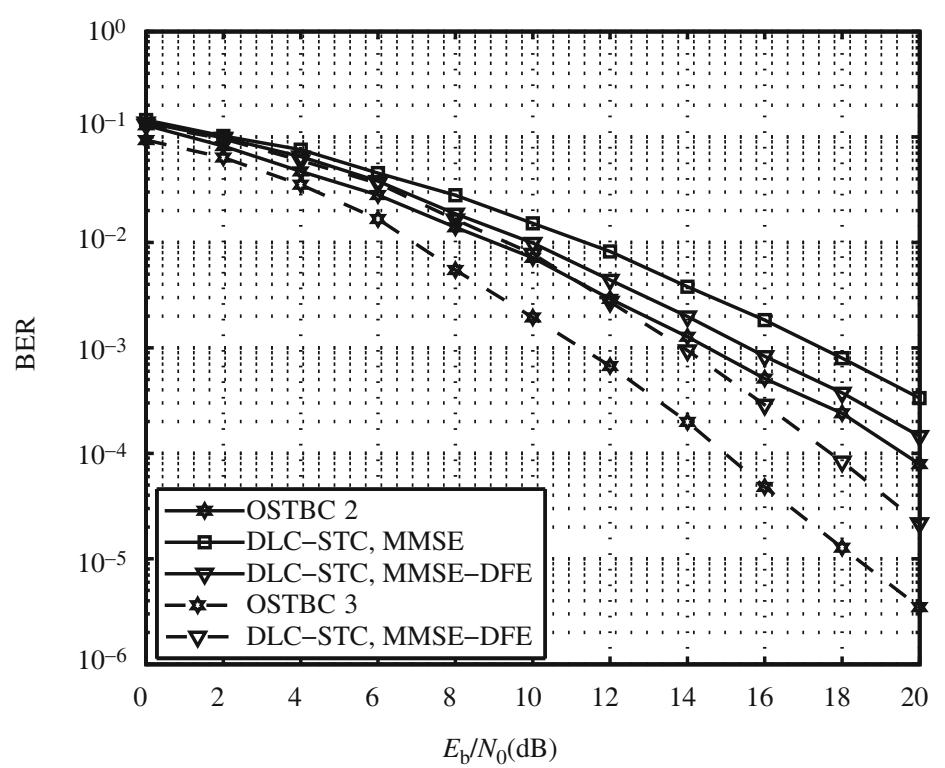

Figure 4 Performances of some examples of DLC-STC proposed in [40] under time asynchronous case. The solid lines are cases where $R=2$, and the dashed lines are $R=3$. QPSK constellation is used, and MMSE and MMSE-DFE receivers are adopted, respectively. The performances of OSTBCs with the same diversity order and perfect time synchronization are also illustrated to show that full cooperative diversity is indeed achieved.

asynchronous code matrix (3) such that it is always full row rank whatever row of the code matrix shifts. For example, the following two matrices satisfy this property:

$$
M_{2}=\left[\begin{array}{ll}
1 & 0 \\
1 & 1
\end{array}\right], \quad M_{3}=\left[\begin{array}{llll}
1 & 0 & 0 & 0 \\
1 & 1 & 0 & 0 \\
1 & 1 & 1 & 1
\end{array}\right]
$$

Further in [35, 36], Shang and Xia develop the theory in [34] and propose the concept of shift full rank (SFR) matrix to name the above property of the STC matrix in (3). Systematic constructions of such a family of codes are also investigated and obtained. Later, using the tool of algebraic number theory, delay-tolerant distributed threaded algebraic space-time (D-TAST) block codes are proposed in [37, 38], which is similar to the TAST code in the MIMO case [50]. The term delay-tolerant code means that the code has the full diversity property under any delay profile. The construction of delay-tolerant TAST codes is also to choose a suitable algebraic number for each layer so that different layers are laid in different algebraic spaces. It is shown in [39] that all the above DSTTC can still maintain the full cooperative diversity when the delays from the cooperative nodes are fractional delays of symbol duration, without the synchronization assumption. In [40], the binary DSTTC studied in [34, 35] are generalized to complex-valued linear convolutive trellis codes, and a family of distributed linear convolutive space time codes (DLC-STC) is proposed that can achieve full cooperative diversity even with the MMSE-DFE receiver $^{2)}$, without the synchronization assumption. Figure 4 shows the performances of some DLC-STC examples. Perfectly time synchronized orthogonal STBC (OSTBC) with the same diversity orders are also demonstrated to show that full diversity is indeed achieved with MMSE or MMSE-DFE receivers. The idea is generalized to frequency-selective fading channels, and another family of DLC-STC is proposed in [41] for achieving both full cooperative diversity and full multipath diversity with linear and DFE receivers.

The frequency domain approach of collecting full cooperative diversity was first proposed in [32] by using Alamouti code for the DF protocal. A simple space-time coding scheme with the OFDM approach

2) Most space-time code designs achieving full diversity are based on ML receiver. STCs achieving full spatial diversity with linear receivers have been studied recently, in [51-54] 


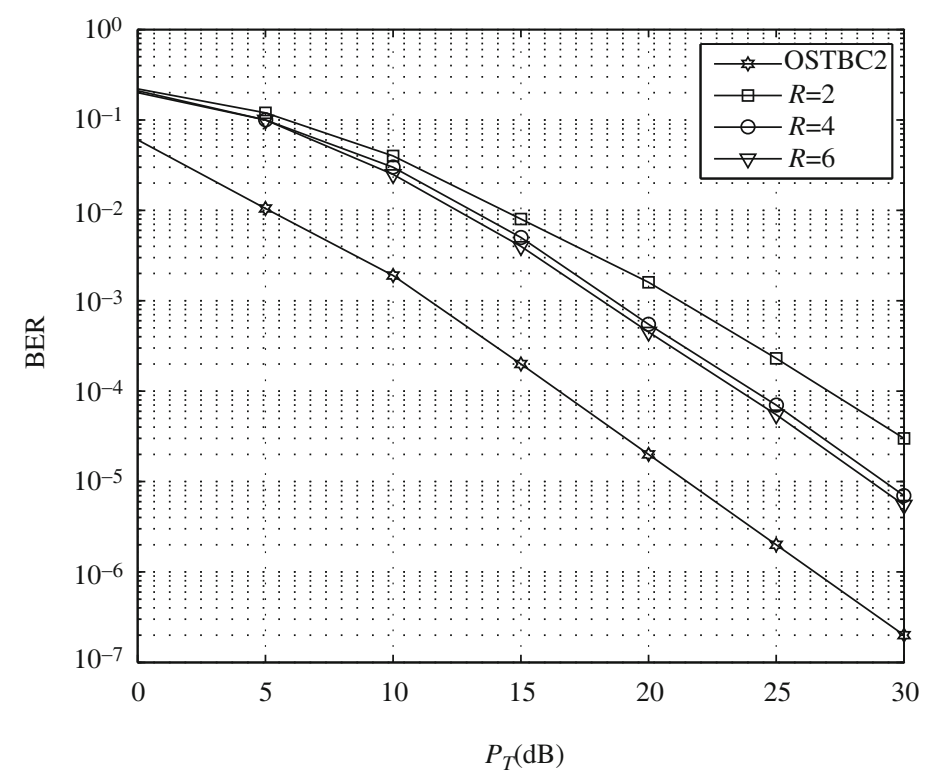

Figure 5 Performances of some examples of DSFC proposed in [42] under time asynchronous case. BPSK constellation is used, and ZF (ML) receiver is adopted.

Table 1 Processing at the cooperative nodes $[42,46]$

\begin{tabular}{ccc}
\hline & $\mathbb{R}_{1}$ & $\mathbb{R}_{2}$ \\
\hline Block 1 & $\alpha_{1} \zeta\left(\boldsymbol{Y}_{11}\right)$ & $-\alpha_{2} \boldsymbol{Y}_{22}^{*}$ \\
Block 2 & $\alpha_{1} \zeta\left(\boldsymbol{Y}_{12}\right)$ & $\alpha_{2} \boldsymbol{Y}_{21}^{*}$ \\
\hline
\end{tabular}

is proposed in [42] for the AF protocal where the cooperative nodes only need to implement some very simple operations and at the destination node, Alamouti code structure can be obtained so that it has fast decoding and achieves full cooperative diversity. The basic idea can be briefly described as follows. At the source node, two consecutive OFDM blocks/symbols $\overline{\boldsymbol{X}}_{1}$ and $\overline{\boldsymbol{X}}_{2}$ of length $N$ together with a sufficient length CP $L_{\mathrm{cp}}$ are transmitted. Denote the $j$-th block received at the cooperative node $i$ by $\boldsymbol{Y}_{i, j}$ of length $L_{s}=N+L_{\mathrm{cp}}$. Then the cooperative nodes will process and transmit the received noisy signals as shown in Table I, where $\alpha_{i}$ is the average transmit power constraint of node $i, i=1,2,(\cdot)^{*}$ denotes the complex conjugation, and $\zeta(\cdot)$ represents the time-reversal of the signal, i.e., $\zeta(\boldsymbol{Y}(n))=\boldsymbol{Y}\left(L_{s}-n\right)$, $n=0,1, \ldots, L_{y}-1$, with $\boldsymbol{Y}\left(L_{s}\right)=\boldsymbol{Y}(0)$. At the destination node, after CP removal for each OFDM block, shifting the last $L_{c p}$ samples of the second OFDM block vector to its beginning, and taking the discrete Fourier transform (DFT), [42] shows that the orthogonality between the subcarriers and the Alamouti code structure are still maintained, which leads to the full diversity [55] and simple ML decoding. For more than two cooperative nodes, [42] proposes to cluster the cooperative nodes into two clusters, treat each cluster as a big node and use the proposed simple coding scheme ${ }^{3)}$. Figure 5 shows the performances of the scheme. In [43], the idea of four group decodable code is exploited so that it is extended to the case of any number of cooperative nodes. In [44], a DSFC based on a layered structure similar to universal space-time codes in [56] has been proposed, which is using the tool of algebraic number theory.

The schemes proposed in [42-44] are for flat fading channels. Under frequency-selective fading scenarios, [46] generalizes the idea in [42] and proposes a simple orthogonal STBC (OSTBC) coded system where the cooperative nodes work in the same way as (or similarly) [42]. Furthermore, with the space-timefrequency coding idea by repeating across the subcarriers [57], [46] proposes a simple space-time-frequency coded scheme that can collect both full cooperative diversity and full multipath diversity from frequencyselective fading channels. With the DF protocal, a high rate space-frequency coding is proposed in [47] to achieve full cooperative and multipath diversities without the synchronization assumption for the cooperative nodes.

3) However, in this case the full cooperative diversity can not be achieved 


\section{$3.2 \quad$ Frequency asynchronism}

From (5), we have already seen that the multiple CFOs make the equivalent channel time-varying. To overcome this, most works are on equalization techniques for the destination node [58-64]. For example, an MMSE-DFE equalizer is designed in [59] to compensate for multiple CFOs at the destination node for the cooperative network adopting delay diversity protocol [30]. In [60, 62], hybrid equalizers with the structure of a linear receiver followed by an ML detector have been proposed for cooperative system adopting DSFC proposed in [47]. However, the time-variant equivalent channel results in a time-varying equalizer. Consequently, the equalizer should be updated again and again; therefore the computational complexity is rather high, generally in the order of $\mathcal{O}\left(N^{4}\right)$ or even larger. Computationally efficient recursive MMSE and MMSE-DFE equalizers are designed in [63] to reduce the computational complexity of the direct equalizations, where large matrix inversion does not need to be updated every block. The recursion idea is also generalized to Kalman equalizer case in [64]. A major drawback for all the above equalizations is that the full diversity gain may not be achieved at the destination node.

To further improve the performance, different DSFC schemes based on the OFDM technique are proposed for the cooperative nodes in [65, 66]. In [65], a cooperative transmission scheme combining intercarrier interference (ICI) self-cancellation precoding with Alamouti STC for two cooperative nodes is proposed. The ICI self-cancellation is first proposed in [67] for the conventional OFDM systems to combat $\mathrm{CFO}$, where only half of the subcarriers are used for data. In [66], another OFDM technique is proposed for overcoming frequency offsets, where longer than OFDM block size CP is required. The decoding complexities of these schemes in $[65,66]$ are very low. However, neither of them is bandwidth efficient. The bandwidth efficiency of [66] is upper bounded by only $1 / R$. To overcome this problem, in [68], two DSFCs, called frequency-reversal SFC (FR-SFC) and frequency-shift SFC (FS-SFC), respectively, are proposed for frequency asynchronous cooperative communications with flat fading channels. The basic motivation is that a flat fading channel with CFO in the time domain can be transformed to a block time-invariant ISI channel in the frequency domain. To show this, take $R=1$ for example. Rewriting (4) into vector-matrix form, and transforming it to the frequency-domain via DFT, we get [68]

$$
\boldsymbol{y}_{f}=h \boldsymbol{F} \boldsymbol{E}(\phi) \boldsymbol{F}^{H} \boldsymbol{x}_{f}+\boldsymbol{w}_{f}=h \overline{\boldsymbol{T}} \boldsymbol{x}_{f}+\boldsymbol{w}_{f},
$$

where $\boldsymbol{F}$ is the $N \times N$ DFT matrix, $\boldsymbol{x}_{f}$ and $\boldsymbol{w}_{f}$ are the frequency domain transmitted data and additive noise, respectively (subscript ${ }_{f}$ denotes the data in the frequency-domain). $\boldsymbol{E}(\phi) \triangleq \operatorname{diag}\left(1, \mathrm{e}^{\mathrm{j} \frac{2 \pi \phi}{N}}, \ldots\right.$, $\mathrm{e}^{\mathrm{j} \frac{2 \pi(N-1) \phi}{N}}$ ), and $\phi$ is the normalized CFO (NCFO) by block duration $T_{b}$, which is defined as $\phi \triangleq \triangle f T_{n}$. $\overline{\boldsymbol{T}}$ is defined as $\overline{\boldsymbol{T}} \triangleq \boldsymbol{F} \boldsymbol{E}(\phi) \boldsymbol{F}^{\mathrm{H}}$, which can be called inter-carrier interference (ICI) matrix since when $\phi \neq 0, \bar{T}$ is not a diagonal matrix and the non-diagonal elements of $\bar{T}$ are the ICI items, or ISI channel matrix. Based on the model of (15) and the properties of ISI channel matrix $\overline{\boldsymbol{T}}$, these codes possess two interesting features: a) Even under the frequency-asynchronous scenario, the codes can achieve full cooperative diversity; b) only linear receivers, such as zero-forcing (ZF) and MMSE receivers, are required to collect the full cooperative diversity, instead of the computationally exhausted ML detector. These two codes achieve a reasonable tradeoff between performance, bandwidth efficiency and decoding complexity, when the number of cooperative nodes is small.

The very recent work [69] generalizes the idea of [68] together with [53] to the network with any number of cooperative nodes, and proposes a systematic design scheme for a family of DSFCs that can achieve full cooperative (spatial) diversity with only linear receivers. The codes have the special structures such that the columns of each code matrix can be divided into several orthogonal groups and each group is stacked by several sub-blocks with Toeplitz structure. The codes in this family have different symbol rates, orthogonalities and performances for different numbers of cooperative nodes, which can be adjusted by choosing different design parameters. In Figure 6, we illustrate the performances of some examples of the family of codes. Perfectly synchronized OSTBCs with the same diversity orders are also demonstrated to show that full diversity is indeed achieved with ZF or MMSE receivers.

A different approach to achieve full diversity in cooperative network with multiple CFOs is proposed in [70], where a diagonal space-frequency code family is proved to maintain the full cooperative diversity 
with the multiple CFOs. However, ML receiver is required to collect the diversity gain, which may be computationally complex.

When both time and frequency asynchronism exist, the schemes in [65, 66] can also be applied, since they both utilize the CP of OFDM system to combat the time offsets. The shortcoming is still the lower bandwidth efficiency. The equalization methods in [58-61, 63] apply as well, but full diversity gain is lost.

\section{Two-way cooperative network with asynchronous cooperative diversity}

We now briefly overview the asynchronous cooperative diversity for two-way cooperative networks.

\subsection{Time asynchronism}

To combat the time asynchronism for two-way cooperative network, both DSTCs and DSFCs are proposed in the time-domain and the frequency domain, respectively. For time domain approaches, in [29], it has been shown that under the equivalent STC model of (11) and (12), a scheme similar to that in [40] can also achieve full cooperative diversity with linear or DFE receivers, as depicted in Figure 7. Since (11) is an AF protocol based model, this implies that even under AF protocol where the noise at cooperative nodes are also amplified and received at the destination, shift full rank property and linear receiver achieving full diversity still hold. This observation actually can raise many new code designs robust to time asynchronism for two-way cooperative network. Another DSTC approach proposed in the timedomain for frequency-selective channels is in [71], which is an extension of [41] from one-way cooperative network to the two-way case. For frequency domain approaches, in [28], the idea in [42] is utilized. OSTBC is combined with the OFDM technique, and CP is used at both source and cooperative nodes to eliminate the time offsets. The low complexity decoding of OSTBC is maintained. It also applies to frequency-selective channels and achieves the frequency (multipath) diversity gain by using the spacetime-frequency coding technique. A difference between [28] (when a one-way network is treated as a special two-way network) and [46] is that no CP needs to be added for cooperative nodes in [46] while it does in [28].

Except for these DSTCs and DSFCs, some binary-field coding schemes are proposed in [72, 73]. BPSK signal is considered in [72], and the penalties of time and frequency synchronization errors are analyzed. A linear convolutional channel coding together with network coding is proposed in [73], which can relax the time synchronization requirement. However, neither of them considered the diversity issue.

\subsection{Frequency asynchronism}

So far, to our best knowledge, there is no literature discussing how to combat frequency asynchronism for a two-way cooperative network. However, from the model of (13), one can see that DSTCs or DSFCs are still efficient approaches to achieve cooperative diversity. The difficulty lies in the fact that the equivalent DSTC matrix $\boldsymbol{X}_{f}^{a}$ that combines the CFOs from $\mathbb{T}_{2}$ to $\mathbb{R}_{r}$, and $\mathbb{R}_{r}$ to $\mathbb{T}_{1}$ is time varying. How to design these codes and the corresponding detection algorithms needs to be addressed in the near future.

\section{Conclusions}

Cooperative communication technology is an efficient way to provide spatial diversity gain to wireless terminals. It can be considered as a distributed MIMO system. Due to its distributed nature, the system may be neither time nor frequency synchronized. In this paper, we have provided a survey of the recent research efforts on combating the time and frequency asynchronism of the cooperative communication network. Our focus is on coding/modulation/signal design and signal processing point of view. We have reviewed various distributed STCs and SFCs and equalization algorithms proposed in the literatures. 


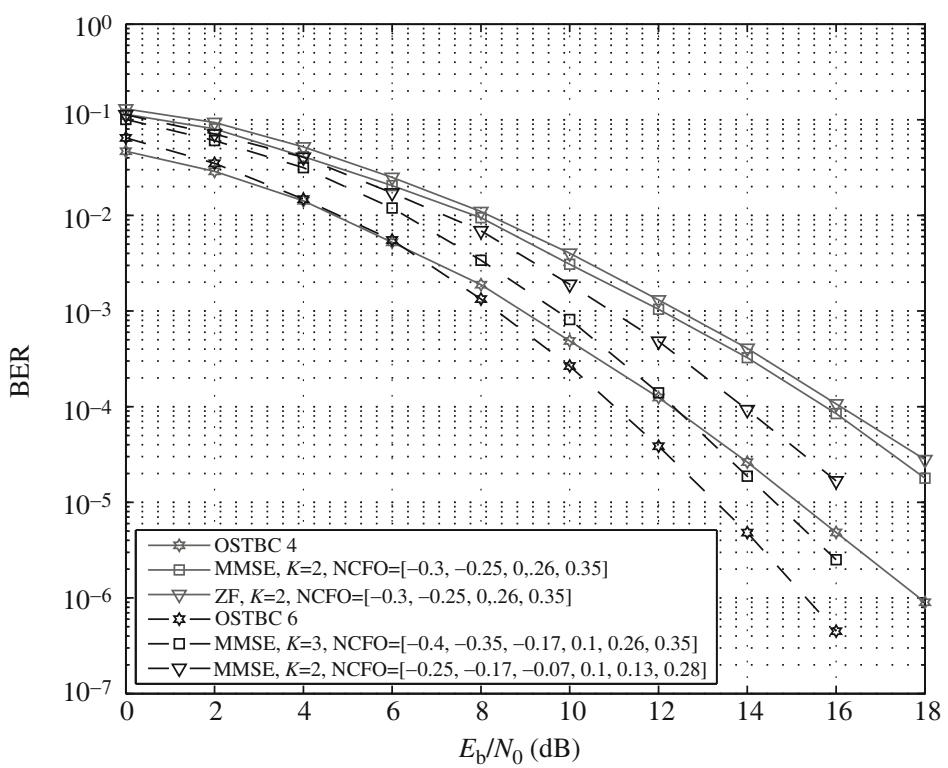

Figure 6 Performances of some examples of the family of codes proposed in [69] under frequency asynchronous case with different NCFOs. The solid lines are cases where $R=4$, and the dashed lines are $R=6$. QPSK constellation is used, and $\mathrm{ZF}$ and MMSE receiver are adopted, respectively. $K$ is a design parameter defined in [69] associated with the number of orthogonal groups. The performances of OSTBCs with the same diversity orders and perfect frequency synchronization are also illustrated to show that full cooperative diversity is indeed achieved.

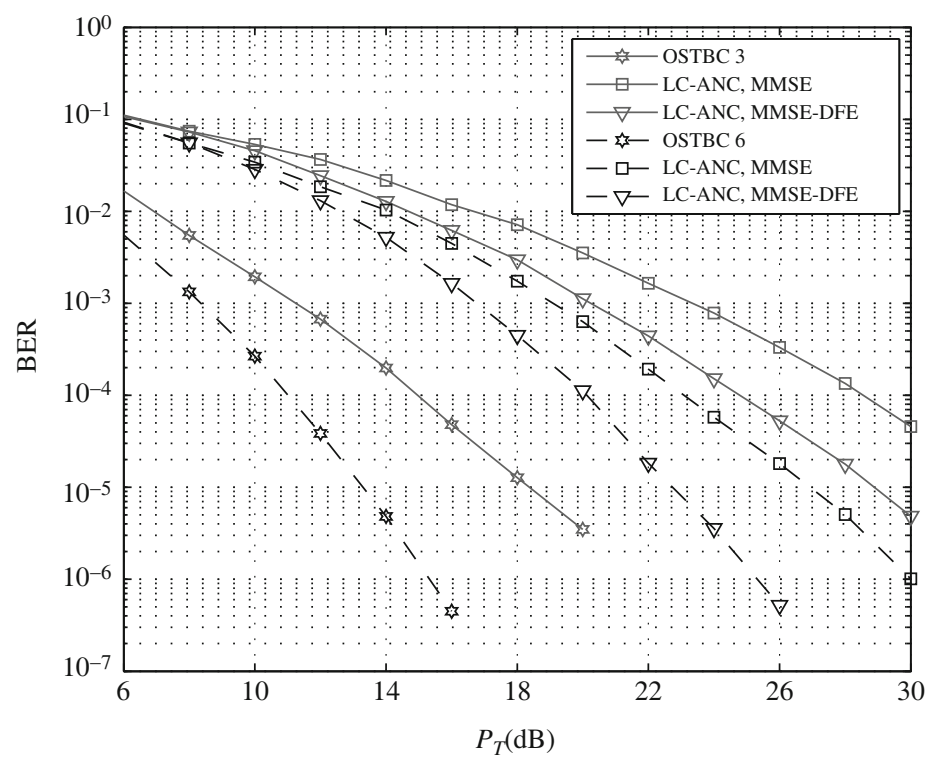

Figure 7 Performances of some examples of linear convolutive analog network coding (LC-ANC) proposed in [29] under time asynchronous case. The solid lines are cases where $R=3$, and the dashed lines are $R=6$. QPSK constellation is used, and MMSE and MMSE-DFE receivers are adopted, respectively. The performances of OSTBCs with the same diversity orders and perfect time synchronization are also illustrated to show that full cooperative diversity is indeed achieved.

Both one-way and two-way cooperative networks are considered. One can see the aim of all these proposed schemes is to achieve a better tradeoff among performance (diversity gain), symbol rate (bandwidth efficiency), and computational complexity. Some future studies may lie in the following aspects.

On the frequency asynchronism of a two-way cooperative network, as we mentioned above, the difficulty of the code design lies in the fact that the equivalent DSTC matrix $\boldsymbol{X}_{f}^{a}$ that combines the CFOs between terminals and cooperative nodes is time-varying. How to design these codes and corresponding detection algorithms needs to be studied further. 
We have only considered the case where the transmitters do not have the CSI. Without CSI, STCs are utilized to collect diversity gain. If feedback is available, another efficient way to provide performance enhancement is beamforming. Recently, there are already some studies on the analysis and design of distributed beamformer for cooperative network when the feedback information is not accurate. However, they only take the channel fading coefficients into account. If time and frequency asynchronism are considered, the performance analysis and new beamformer design should be re-investigated.

Finally, as we mentioned at the beginning, except for the synchronization issue, signal coordination is another practical problem caused by the distributed nature. All the literatures we have overviewed made the assumption that the cooperative network is controlled by some central station and it has already been coordinated by some high-layer protocol However, in many applications like wireless ad hoc network or WSN, there is no such control station. Therefore, from a physical-layer point of view, how to design codes that rely on coordination as less as possible is an interesting point. In [74], the authors have proposed a random STC to release the coordination. More practical code design is still an open problem.

\section{Acknowledgements}

This work was supported by the National Natural Science Foundation of China (Grants No. 61071125, 60971113), the Research Fund for Doctoral Program of Higher Education of China (Grant No. 200806980020), the Science Foundation for Innovation Research Group of China (Grant No. 60921003), the National Science Foundation (NSF) (Grant No. CCF-0964500), the World Class University (WCU) Program, National Research Foundation, Korea.

\section{References}

1 Telatar E. Capacity of multi-antenna Gaussian channels. European Trans Telecom, 1999, 10: 585-595

2 Foschini G J, Gans M J. On limits of wireless communications in a fading environment when using multiple antennas. Wirel Pers Commun, 1998, 6: 311-335

3 Tarokh V, Seshadri N, Calderbank A R. Space-time codes for high data rate wireless communication: Performance criterion and code construction. IEEE Trans Inform Theory, 1998, 44: 744-765

4 Alamouti S M. A simple transmit diversity technique for wireless communications. IEEE J Select Area Commun, 1998, 16: $1451-1458$

5 Foschini G J. Layered space-time architecture for wireless communication in a fading environment when using multielement antennas. Bell Labs Tech J, 1996, 1: 41-59

6 Tarokh V, Jafarkhani H, Calderbank A R. Space-time block codes from orthogoal designs. IEEE Trans Inform Theory, 1999, 45: 1456-1467

7 Sendonaris A, Erkip E, Aazhang B. User cooperative diversity - Part I: System description, Part II: Implementation aspects and performance analysis. IEEE Trans Commun, 2003, 51: 1927-1948

8 Laneman J N, Tse D, Wornell G. Cooperative diversity in wireless networks: Efficient protocols and outage behavior. IEEE Trans Inform Theory, 2004, 50: 3062-3080

9 Laneman J, N, Wornell W. Distributed space-time-coded protocols for exploiting cooperative diversity in wireless networks. IEEE Trans Inform Theory, 2003, 49: 2415-2425

10 Janani M, Hedayat A, Hunter T E, et al. Coded cooperation in wireless communications: Space-time transmission and iterative decoding. IEEE Trans Signal Process, 2004, 52: 362-371

11 Stefanov A, Erkip E. Cooperative space-time coding for wireless networks. IEEE Trans Commun, 2005, 53: 1804-1809

12 Jing Y, Hassibi B. Distributed space-time coding in wireless relay networks. IEEE Trans Wirel Commun, 2006, 5: $3524-3536$

13 Pabst R, Walke B H, Schultz D C, et al. Relay-based deployment concepts for wireless and mobile broadband radio. IEEE Commun Mag, 2004, 42: 80-89

14 Scaglione A, Goeckel D L, Laneman J N. Cooperative communications in mobile ad hoc networks: Rethinking the link abstraction. IEEE Signal Process Mag, 2006, 23: 18-29

15 Stankovic V, Host-Madsen A, Xiong Z. Cooperative diversity for wireless ad hoc networks: capacity bounds and code designs. IEEE Signal Process Mag, 2006, 23: 37-49

16 Wei S. Diversity-multiplexing tradeoff of asynchronous cooperative diversity in wireless networks. IEEE Trans Inform Theory, 2007, 53: 4150-4172 
17 Kuo W Y, Fitz M P. Frequency offset compensation of pilot symbol assisted modulation in frequency flat fading. IEEE Trans Commun, 1997, 45: 1412-1416

18 Iltis R A, Mirzaei S, Kastner R. Carrier offset and channel estimation for cooperative MIMO sensor networks. In: Proc IEEE Globecom, San Francisco, 2006. 1-5

19 Pham T H, Nallanathan A, Liang Y C. Joint channel and frequency offset estimation in distributed MIMO flat-fading channels. IEEE Trans Wireless Commun, 2008, 7: 648-656

20 Parker P A, Mitran P, Bliss B W, et al, On bounds and algorithms for frequency synchronization for collaborative communication systems. IEEE Trans Signal Process, 2008, 56: 3742-3752

$21 \mathrm{Li} \mathrm{X}, \mathrm{Wu}$ Y C, Serpedin E. Timing synchronization in decode-and-forward cooperative communication systems. IEEE Trans Signal Process, 2009, 57: 1444-1455

22 Wang J Z, Milstein L B. CDMA overlay situations for microcellular mobile communications. IEEE Trans Commun, 1995, COM-43: 603-614

23 Rankov B, Wittneben A. Achievable rate regions for the two-way relay channel. In: Proc IEEE International Symp Inform Theory (ISIT), 2006. 1668-1672

24 Rankov B, Wittneben A. Spectral efficient protocols for halfduplex fading relay channels. IEEE J Sel Area Commun, 2007, 25: 379-389

25 Katti S, Gollakota S, Katabi D. Embracing wireless interference: analog network coding. In: Proc ACM SIGCOMM, 2007. 397-408

26 Zhang S, Liew S C, Lam P P. Physical layer network coding. In: Proc MobiCOM, 2006. 358-365

27 Koike-Akino T, Popovski P, Tarokh V. Optimized constellation for two-way wireless relaying with physical network coding. IEEE J Sel Areas Commun, 2009, 27: 773-787

28 Li Z, Xia X G, Li B. Achieving full diversity and fast ML decoding via simple analog network coding for asynchronous two-way relay networks. IEEE Trans Commun, 2009, 57: 3672-3681

29 Wang H M, Xia X G, Yin Q. A linear analog network coding for asynchronous two-way relay networks. IEEE Trans Wireless Commun, 2010, 9: 3630-3637

30 Wei S, Goeckel D, Valenti M. Asynchronous cooperative diversity. IEEE Trans Wireless Commun, 2006, 5: 1547-1557

31 Li X. Space-time coded multi-transmission among distributed transmitters without perfect synchronization. IEEE Signal Process Lett, 2004, 11: 948-951

32 Mei Y, Hua Y, Swami A, et al. Combating synchronization errors in cooperative relays. In: Proc IEEE ICASSP, Vol 3 , 2005. 369-372

33 Zheng F C, Burr A G, Olafsson S. Near-optimum detection for distributed space-time block coding under imperfect synchronization. IEEE Trans Commun, 2008, 56: 1795-1799

34 Li Y, Xia X G. A family of distributed space-time trellis codes with asynchronous cooperative diversity. IEEE Trans Commun, 2007, 55: 790-800. Partially presented in Proc the Fourth International Conf Information Processing in Sensor Networks (IPSN), UCLA, Los Angeles: 2005. 25-27

35 Shang Y, Xia X G. Shift full rank matrices and applications in space-time trellis codes for relay networks with asynchronous cooperative diversity. IEEE Trans Inform Theory, 2006, 52: 3153-3167

36 Shang Y, Xia X G. Limited-shift-full-rank matrices with applications in asynchronous cooperative communications. IEEE Trans Inform Theory, 2007, 53: 4199-4126

37 Hammons A R. Algebraic space-time codes for quasi-synchronous cooperative diversity. In: Proceedings IEEE Conference Wireless Networks, Commun Mobile Comput, Maui, HI, 2005. 11-15

38 Damen M O, Hammons A R. Delay-tolerant distributed TAST codes for cooperative diversity. IEEE Trans Inform Theory, 2007, 53: 3755-3773

39 Shang Y, Xia X G. Space-time trellis codes with asynchronous full diversity up to fractional symbol delays. IEEE Trans Wirel Commun, 2008, 7: 2473-2479

40 Guo X, Xia X G. Distributed linear convolutive space-time codes for asynchronous cooperative communication networks. IEEE Trans Wirel Commun, 2008, 7: 1857-1861

41 Zhong Z, Zhu S, Nallanathan A. Delay-tolerant distributed linear convolutional space-time code with minimum memory length under frequency-selective channels. IEEE Trans Wirel Commun, 2009, 8: 3944-3949

42 Li Z, Xia X G. A simple Alamouti space-time transmission scheme for asynchronous cooperative systems. IEEE Signal Process Lett, 2007, 14: 804-807

43 Rajan G S, Rajan B S. OFDM based distributed space time coding for asynchronous relay networks. In: Proc IEEE Int Conf Commun (ICC), 2008. 1118-1122

44 Guo X, Xia X G. A distributed space-time coding in asynchronous wireless relay networks. IEEE Trans Wirel Commun, 2008, 7: 1812-1816

$45 \mathrm{Lu} \mathrm{H} \mathrm{F}$. Constructions of fully-diverse high-rate space-frequency codes for asynchronous cooperative relay networks. In: Proc IEEE International Symposium of Information Theory (ISIT). Toronto, 2008. 832-836

46 Li Z, Xia X G, Lee M H. A simple orthogonal space-time coding scheme for asynchronous cooperative systems for frequency selective fading channels. IEEE Trans Commun, 2010, 58: 2219-2224. Also downloadable at http://www.ee.udel. 
edu/ xxia/Pub.html/paper_li_xia_2010.pdf and http://www.ee.udel.edu/ xxia/Pub.html/correction_li_xia.pdf

47 Li Y, Zhang W, Xia X G. Distributive high-rate space-frequency codes achieving full cooperative and multipath diversities for asynchronous cooperative communications. IEEE Trans Veh Technol, 2009, 58: 207-217

48 Wittneben A. Base station modulation diversity for digital SIMULCAST. In: Proc IEEE VTC'93, 1993. 505-511

49 Lindskog E, Paulraj A. A transmit diversity scheme for channels with intersymbol interference. In: Proc IEEE ICC'00. New Orleans, 2000. 307-311

50 Damen M O, Gamal H E, Beaulieu N C. Systematic construction of full algebraic diversity constellations. IEEE Trans Inform Theory, 2003, 49: 3344-3349

51 Liu J, Zhang J K, Wong M. Full-diversity codes for MISO systems equipped with linear or ML detectors. IEEE Trans Inform Theory, 2008, 54: 4511-4527

52 Shang Y, Xia X G. On space-time block codes achieving full diversity with linear receivers. IEEE Trans Inform Theory, 2008, 54: 4528-4547

53 Wang H, Xia X G, Yin Q, et al. A family of space-time block codes achieving full diversity with linear receivers. IEEE Trans Commun, 2009, 57: 3607-3617

54 Zhang W, Yuan J. A simple design of space-time block codes achieving full diversity with linear receivers. In: Proc ICASSP. Taipei, 2009. 2729-2732

55 Zhang S B, Xia X G, Wang J Z. Cooperative performance and diversity gain of wireless relay networks (in press). Partially appeared in Proc IEEE VTC2011-Spring, Budapest, 2011. 15-18

56 Gamal H E, Damen M O. Universal space-time coding. IEEE Trans Inform Theory, 2003, 49: 1097-1119

57 Zhang W, Xia X G, Ching P C. Full-diversity and fast ML decoding properties of general orthogonal space-time block codes for MIMO-OFDM systems. IEEE Trans Wirel Commun, 2007, 6: 1647-1653

58 Li Z, Qu D, Zhu G. An equalization technique for distributed STBC-OFDM system with multiple carrier frequency offsets. In: Proc IEEE WCNC, vol 2, 2006. 839-843

59 Veronesi D, Goeckel D L. Multiple frequency offset compensation in cooperative wireless systems. In: Proc IEEE Globecom'06. San Francisco, 2006. 1-5

60 Tian F, Xia X G, Ching P C. Signal detection for space-frequency coded cooperative communication system with multiple carrier frequency offsets. In: Proc IEEE Wireless Communications Networking Conf (WCNC). Hong Kong, 2007. 1221-1225

61 Benvenuto N, Tomasin S, Veronesi D. Multiple frequency offsets estimation and compensation for cooeperative networks. In: Proc IEEE WCNC'07. Hong Kong, 2007. 892-896

62 Tian F, Xia X G, Ching P C, et al. Signal detection in a space-frequency coded cooperative communication system with multiple carrier frequency offsets by exploiting specific properties of the code structure. IEEE Trans Veh Technol, 2009, 58: 3396-3409

63 Wang H, Xia X G, Yin Q. Computationally efficient equalization for asynchronous cooperative communications with multiple frequency offsets. IEEE Trans Wirel Commun, 2009, 8: 648-655

64 Wang H M, Yin Q, Xia X G. Fast Kalman equalization for time-frequency asynchronous cooperative relay networks with distributed space-time codes. IEEE Trans Veh Technol, 2010, 59: 4651-4658

65 Li Z, Xia X G. An Alamouti coded OFDM transmission for cooperative systems robust to both timing errors and frequency offsets. IEEE Trans Wirel Commun, Part II, 2008, 7: 1839-1844

$66 \mathrm{Li} \mathrm{X}, \mathrm{Ng} \mathrm{F}$, Han T. Carrier frequency offset mitigation in asynchronous cooperative OFDM transmissions. IEEE Trans Signal Process, 2008, 56: 675-685

67 Zhao Y, Haggman S G. Intercarrier interference self-cancellation scheme for OFDM mobile communication systems. IEEE Trans Commun, 2001, 49: 1185-1191

68 Wang H, Xia X G, Yin Q. Distributed space-frequency codes for cooperative communication systems with multiple carrier frequency offsets. IEEE Trans Wirel Commun, 2009, 8: 1045-1055

69 Wang H, Yin Q, Xia X G. Full diversity space-frequency codes for frequency asynchronous cooperative relay networks with linear receivers. IEEE Trans Commun, 2011, 59: 236-247

70 Tian F, Xia X G, Ma K, et al. On the full diversity property of a space-frequency code family with multiple frequency offsets in cooperative communication systems. J Commun, 2010, 5: 317-331

71 Zhong Z, Zhu S, Lü G. Distributed space-time code for asynchronous two-way wireless relay networks under frequencyselective channels. In: Proc IEEE International Conf Commun (ICC). Dresten, 2009. 1-5

72 Zhang S, Liew S C, Lam P P. On the synchronization of physical-layer network coding. In: Proc IEEE Inform Theory Workshop (ITW), 2006. 404-408

73 Wang D, Fu S, Lu K. Channel coding design to support asychronous phasical layer network coding. In: Proc IEEE Globecom'09. Honolulu, HI, 2009. 1-6

74 Sirkeci-Mergen B, Scaglione A. Randomized space-time coding for distributed cooperative communication. IEEE Trans Signal Process, 2007, 55: 5003-5017 